\title{
Development of Bioadhesive Microspheres for Oral Bioavailability Enhancement of Berberine Hydrochloride
}

\author{
Ye Zhang' and Hongming Liu ${ }^{2}$ \\ ${ }^{1}$ Department of Pharmaceutical Sciences, Zibo Vocational Institute, Zibo, Shandong 255314, China \\ ${ }^{2}$ Zibo Institute for Food and Drug Control, Zibo, Shandong 255086, China \\ Correspondence should be addressed to Ye Zhang; yezhangye2006@126.com
}

Received 22 May 2016; Accepted 20 September 2016

Academic Editor: Ruilong Sheng

Copyright (c) 2016 Y. Zhang and H. Liu. This is an open access article distributed under the Creative Commons Attribution License, which permits unrestricted use, distribution, and reproduction in any medium, provided the original work is properly cited.

\begin{abstract}
The objective of this study was to innovatively prepare chitosan-coated alginate/gelatin BBH loaded microspheres and evaluate their pharmaceutical characteristics and pharmacokinetics. The bioadhesive microspheres were prepared using an emulsification technique. Three batches of microspheres were formed and their stability was evaluated. BBH loaded microspheres were almost spherical with shallow elevation on surfaces. The mean particle size of microspheres was $368.2 \mu \mathrm{m}$, drug loading was $3.59 \pm 0.01 \%$, and in situ bioadhesion percentage was $91.23 \% \pm 8.2 \%$ and they achieved a sustained release with $71.29 \%$ for 8 hours in vitro. Pharmacokinetic studies in rats indicated that the bioavailability of BBH microspheres was enhanced about 1.5-fold as compared with commercial tablets. BBH microspheres exhibited a sustained-release profile over $48 \mathrm{~h}$. Thus, chitosan-coated alginate/gelatin $\mathrm{BBH}$ loaded microspheres which combined the advantages of alginate/gelatin microspheres and chitosan may be used as a sustained delivery system for BBH to treat duodenal and benign gastric ulcers.
\end{abstract}

\section{Introduction}

Berberine hydrochloride $(\mathrm{BBH})$, an active isoquinoline alkaloid, is widely present in various traditional Chinese medicines such as Hydrastis canadensis (goldenseal), Coptis chinensis (Coptis or golden thread), Berberis aquifolium (Oregon grape), Berberis vulgaris (barberry), and Berberis aristata (tree turmeric). BBH is commercially used to treat duodenal and benign gastric ulcers caused by bacteria. $\mathrm{BBH}$ has been found to be effective for diabetes and obesity, partly via stimulating AMP-activated protein kinase activity $[1,2]$. BBH has also shown antidepressant activity by modulating brain biogenic amines (norepinephrine, serotonin, and dopamine), oxide pathway, and/or sigma receptors [3-5]. Recently, BBH has been reported as a novel cholesterol-lowering agent, and it functions through a unique mechanism distinct from statins [6]. However, $\mathrm{BBH}$, one of the poorly water-soluble drugs and substrate of P-glycoprotein [7, 8], has low mucosal permeability [9], resulting in limited absorption in the gastrointestinal tract, which seriously limits its application and development as a pharmaceutical preparation. Furthermore, intramuscular and intravenous administration of $\mathrm{BBH}$ could result in adverse reactions such as anaphylactic shock and drug eruption [10]. Hence, a novel drug delivery system to improve the solubility and bioavailability of $\mathrm{BBH}$ has drawn great attention of researchers in pharmaceutical industry.

In the last decade, microspheres have prospered enormously because of a variety of applications such as delivery vesicles for drugs, deoxyribonucleic acids, antigens, proteins, and enzymes, especially for controlled or sustained drugdelivering systems employing biopolymers as raw material [11-13]. Recently, in pharmaceutical industry, microspheres have drawn great attention due to their excellent efficiency in prolonging the half-life time of drug and improving bioavailability of drug in vivo by controlling release rate of drug from the microspheres [14-17]. In addition, simple techniques are involved in the preparation of microspheres [18-21]. At present, a couple of biodegradable polymers such as chitosan, alginate, and gelatin have been used to prepare microspheres [22-24]. Chitosan is a type of excellent natural hydrophilic polysaccharide among many biodegradable polymers, and it is nontoxic and shows excellent mucoadhesive and permeation-enhancing effects across biological surfaces [25]. Chitosan exhibits antacid and antiulcer effects, which 
may prevent or weaken drug irritation in the stomach [26]. Additionally, chitosan is a $\mathrm{pH}$-sensitive hydrogel and, at low $\mathrm{pH}$ value, protonated amino group $\left(-\mathrm{NH}_{3}{ }^{+}\right)$can make chitosan molecules adhere to mucosal surfaces easily. Hence, chitosan has great potential in oral drug delivery system to treat gastric ulceration. Alginate has bioadhesive properties and can also be effective in protecting mucous membranes of the gastrointestinal tract. In the present study, a sustained drug delivery system of chitosan-coated alginate/gelatin gastric mucoadhesive $\mathrm{BBH}$ loaded microspheres was designed to treat duodenal and benign gastric ulcers. The microspheres were cross-linked with 1-ethyl-3- (3-dimethylaminopropyl) carbodiimide (EDC) and N-hydroxysuccinimide (NHS). EDC is a condensation agent between carboxyl and amino groups to form amide bonds to immobilize the microspheres and it has lower cytotoxicity compared with other chemical cross-linking agents such as glutaraldehyde and formaldehyde [27]. The microspheres were characterized using scanning electron microscopy (SEM), particle size and distribution analysis, and in situ bioadhesion test. The in vitro dissolution rate of microspheres was measured in simulated gastric fluid by high-performance liquid chromatography (HPLC), and the in vivo bioavailability in rats was assayed using tandem mass spectroscopy coupled to ultraperformance liquid chromatography (UPLC-MS/MS).

\section{Materials and Methods}

2.1. Materials. $\mathrm{BBH}$, chitosan, gelatin, EDC, and NHS were obtained from Sigma-Aldrich Co. (St. Louis, MO, USA). BBH commercial tablets were bought from First Teaching Hospital of Tianjin University of TCM. Span 80 was purchased from Croda International Plc (Yorkshire, UK). Sodium alginate was obtained from Bio Basic Inc. (Toronto, Canada). Liquid paraffin was purchased from Tianjin No. 1 Chemical Reagent Factory (Tianjin, China). All other chemicals were of analytical grade.

\subsection{Preparation of Chitosan-Coated Alginate/Gelatin $B B H$} Loaded Microspheres. BBH loaded microspheres were prepared by water-in-oil emulsion technique as previously reported [28]. Briefly, water phase containing sodium alginate/gelatin $(2: 3)(5.0 \% \mathrm{w}: \mathrm{v})$ and $\mathrm{BBH}(0.5 \mathrm{~g})$ was dissolved in $2 \%(\mathrm{v} / \mathrm{v})$ acetic acid aqueous solution. Oil phase containing liquid paraffin and emulsifiers (Span 80 and Tween 80, 4:1 ratio, v/v) was homogenized under stirred conditions at $40^{\circ} \mathrm{C}$. Later, water phase was added to oil phase $(1: 5, \mathrm{v}: \mathrm{v})$ under stirred conditions of $450 \mathrm{rpm}$ at $40^{\circ} \mathrm{C}$. The prepared mixture was kept over an ice-water bath for approximately $15 \mathrm{~min}$ and then $20 \mathrm{~mL}$ of isopropanol was added to the mixture with continuous stirring for $10 \mathrm{~min}$ to obtain a stable emulsion system. $4 \mathrm{~mL}$ of cross-linking agents (EDC/NHS, $4: 1$, w/w) was dissolved in $50 \mathrm{mM}$ of MES buffer and they were then added to the inverse emulsion with continuous stirring for $1 \mathrm{~h}$ in ice-water bath, followed by stirring for $4 \mathrm{~h}$ in room temperature. Then, $120 \mathrm{~mL}$ of acetone was added to end the cross-linking reaction. The microspheres were collected by filtration. Washing cycles were performed with acetone and isopropanol before redispersion in distilled water and subsequent lyophilization. The prepared microspheres were immersed into the $0.5 \%$ chitosan acetate aqueous solution at $30^{\circ} \mathrm{C}$ for $30 \mathrm{~min}$. Finally, chitosan-coated alginate/gelatin $\mathrm{BBH}$ loaded microspheres were collected and washed three times with deionized water. The microspheres were immersed in carbodiimide solution for $12 \mathrm{~h}$ and they were then dried at $40^{\circ} \mathrm{C}$. Three batches of microspheres were formed.

\subsection{Characterization of Chitosan-Coated Microspheres}

2.3.1. SEM Observation of Microspheres. The shape and surface features of microspheres were observed using SEM (Philips XL30, Netherlands). The microspheres were suspended in distilled water and the dispersion was dropped on glass slide and dried at ambient atmosphere. The samples were coated with gold for SEM observation.

2.3.2. Particle Size and Distribution Analysis. The microspheres were dispersed in distilled water and the particle size and distribution of microspheres were measured by laser diffractometry using LS230 Coulter (Coulter Co., USA).

2.3.3. Drug Entrapment Efficiency and Loading. Drug entrapment efficiency, and loading were determined by dissolving known amount of microspheres in $50 \mathrm{~mL}$ of simulated gastric fluid under stirring for $48 \mathrm{~h}$ at $37^{\circ} \mathrm{C}$. Then, the supernatant was filtered and analyzed using HPLC (Waters 2695 System, Milford, USA). Drug entrapment efficiency and loading were calculated using the following equations:

$$
\begin{aligned}
& \text { Drug entrapment efficiency } \\
& \quad=\frac{\text { actual BBH content }}{\text { theoretical BBH content }} \times 100 \%, \\
& \text { Drug loading }=\frac{\mathrm{BBH} \text { content }}{\text { microcapsules weight }} \times 100 \% .
\end{aligned}
$$

2.3.4. Stability Study. The microspheres were stored at $60 \pm$ $2^{\circ} \mathrm{C}, 92.5 \pm 5 \% \mathrm{RH}$, and $4500 \pm 500 \mathrm{~lx}$ for 10 days without package. Samples were withdrawn at different time and evaluated for appearance and $\mathrm{BBH}$ content.

2.3.5. Bioadhesion of Chitosan-Coated BBH Loaded Microspheres. The bioadhesion of chitosan-coated $\mathrm{BBH}$ loaded microspheres was investigated according to the method previously reported by Rao and Buri [29]. Male Sprague-Dawley rats (weighing 450-500 g) were maintained at standard conditions and fasted overnight with free access to water until experiment. All procedures were performed in accordance with the National Institutes of Health Guide for the Care and Use of Laboratory Animals. The experimental protocol was approved by the Committee on Animal Research of Zibo Institute for Food and Drug Control.

The rats were anaesthetised with 5\% chloral hydrate ( $250 \mathrm{mg} / \mathrm{kg}$, i.p.). Stomach was dissected and rinsed with physiological saline till the mucosa was clean. The tissue was used within $2 \mathrm{~h}$ after dissection. Then, the stomach was cut longitudinally, spread, and placed on a microscope slid. $50 \mathrm{mg}$ 
of uncoated or coated microspheres was placed uniformly on the mucosa of the stomach. The microscope slid was then placed in a desiccator maintained at $>80 \%$ relative humidity and room temperature. After $20 \mathrm{~min}$, the microscope slid was fixed in a washing jar at an angle of $45^{\circ}$. The stomach mucosa was washed for $5 \mathrm{~min}$ with $30 \mathrm{~mL}$ of flushing liquid $(0.9 \% \mathrm{NaCl}, 0.1 \mathrm{~mol} / \mathrm{L} \mathrm{HCl}, \mathrm{pH} 1.2 \pm 0.1)$ at the rate of $0.1 \mathrm{~mL} / \mathrm{s}$ using a peristaltic pump. The washings were collected, dried, and weighed. The bioadhesion of stomach was calculated according to the following equation:

$$
\text { Bioadhesion }(\%)=\frac{M-(G-m)}{M} \times 100 \%,
$$

where $M$ is the quality of microspheres $(50 \mathrm{mg}), G$ is the quality of the dried residue, and $m$ is the quality of the solid in the $30 \mathrm{~mL}$ of flushing liquid.

2.4. In Vitro Release Studies. The dissolution study was performed using rotating basket method (Sotax AT7, Basel, Switzerland). The microspheres and tablets equivalent to $10 \mathrm{mg} \mathrm{BBH}$ were dispersed in $200 \mathrm{~mL}$ of simulated gastric fluid and stirred at $50 \mathrm{rpm}$ at $37 \pm 0.5^{\circ} \mathrm{C}$. A $0.5 \mathrm{~mL}$ of the suspension was withdrawn at specified time intervals, filtered, and the concentration of the supernatant was analyzed using HPLC. $5 \mu \mathrm{L}$ of the collected supernatant was injected into a chromatograph (Waters 2695, USA) equipped with a UV detector (Waters 2487) and a reversed phase ProntoSIL 120-5- $\mathrm{C}_{18}$-ace-APS column $(250 \mathrm{~mm} \times 4.6 \mathrm{~mm}$, $5 \mu \mathrm{m}$, Bischoff Chromatography, Germany). The mobile phase was acetonitrile-water $(30: 70)$ containing $0.1 \%$ of phosphoric acid and $0.05 \%$ of triethylamine. The flow rate was $1.0 \mathrm{~mL} / \mathrm{min}$ and the UV detector was set at a wavelength of $263 \mathrm{~nm}$.

2.5. In Vivo Availability Studies. The bioavailability of microspheres was compared with $\mathrm{BBH}$ commercial tablets. Male Sprague-Dawley rats (weighing 200-250 g) were randomly divided into two groups of twelve rats each. The rats were fasted overnight with free access to water until administered with microspheres (prepared by dispersing the microspheres in distilled water) or $\mathrm{BBH}$ commercial tablets (24 mg BBH/kg).

$0.5 \mathrm{~mL}$ of blood samples was collected from the retinal venous plexus into heparinized Eppendorf tubes at 0.083 , $0.25,0.50,0.75,1,2,3,4,6,8,12$, and $24 \mathrm{~h}$. Blood samples were immediately centrifuged at $5000 \mathrm{rpm}$ for $10 \mathrm{~min}$ and stored at $-20^{\circ} \mathrm{C}$ until UPLC-MS/MS analysis.

Tetrahydropalmatine was used as an internal standard at a final concentration of $2 \mathrm{ng} / \mathrm{mL}$. $200 \mu \mathrm{L}$ of acetonitrilemethanol $(50: 50, \mathrm{v} / \mathrm{v})$ solution was added to $50 \mu \mathrm{L}$ of rat plasma. After vortex mixing for $1 \mathrm{~min}$, the resultant mixture was centrifuged at $15000 \mathrm{rpm}$ for $10 \mathrm{~min}$. The supernatant was transferred to a clean tube and $10 \mu \mathrm{L}$ of supernatant was injected into a Shimadzu LC-20AD system equipped with a binary pump, a micro vacuum degasser, and an autoinjector. The separation was performed on an Agilent ZORBAX XDB-C18 column $(2.1 \times 50 \mathrm{~mm}, 3.5 \mu \mathrm{m})$. The mobile phase consisted of $0.1 \%(\mathrm{v} / \mathrm{v})$ formic acid in water $(\mathrm{A})$ and $0.1 \%(\mathrm{v} / \mathrm{v})$ formic acid in methanol. The flow gradient was as follows:
0-0.6 min, 90\% A; 0.6-1.2 $\mathrm{min}, 90-2 \% \mathrm{~A} ; 1.2-3.0 \mathrm{~min}, 2 \% \mathrm{~A}$; $3.0-3.1 \mathrm{~min}, 2-90 \% \mathrm{~A}$; and $3.1-4.5 \mathrm{~min}, 90 \% \mathrm{~A}$, at a flow rate of $0.45 \mathrm{~mL} / \mathrm{min}$.

The abovementioned UPLC system was connected with an API 4000 QTRAP mass spectrometry system via an ESI interface. The ESI-MS spectra of samples and reference compounds were acquired in positive ionization modes. The set parameters were as follows: the multiple reaction monitoring mode at $m / z 336.10 \rightarrow 292.10(\mathrm{BBH})$ and $\mathrm{m} / z$ $356.3 \rightarrow 192.10$ (tetrahydropalmatine), curtain gas at $20 \mathrm{psi}$, ion spray voltage at $5.0 \mathrm{kV}$, temperature at $500^{\circ} \mathrm{C}$, ion source gas1 at 55 psi, and ion source gas 2 at 55 psi.

The pharmacokinetic parameters, maximum plasma concentration $\left(C_{\max }\right)$, and time to reach $C_{\max }\left(T_{\max }\right)$ were obtained directly from the plasma concentration-time data. The area under the plasma concentration-time curve (AUC) was calculated using the trapezoidal rule. The values of $C_{\max }$ and AUC were analyzed statistically using analysis of variance after logarithmic transformation. A $P$ value $<0.05$ was considered statistically significant. The relative bioavailability of microspheres was calculated using the following equation: $\mathrm{Fr}=\mathrm{AUC}_{\text {microphere }} / \mathrm{AUC}_{\text {reference(commercial tablets) }}$.

\section{Results}

SEM photographs showed that particles of alginate/gelatin $\mathrm{BBH}$ loaded microspheres with deep elevation on surfaces were spherical on the whole (Figure 1(a)). However, after coating with chitosan, the microcapsules were almost spherical with shallow elevation on surfaces (Figure 1(b)). This morphological characterization may be due to ice crystal sublimation in the process of lyophilization, leading to porous sponginess-like surface. This could ensure microspheres with good fluidity and large specific surface, leading to improved drug absorption. Particle distribution of $\mathrm{BBH}$ microspheres is shown in Figure 2. The median diameter before and after coating with chitosan was $302.0 \mu \mathrm{m}$ and $368.2 \mu \mathrm{m}$, respectively. This increase of $66.2 \mu \mathrm{m}$ was considered to be due to the thickness of the coating layer. These two microspheres displayed Gaussian distribution. The entrapment efficiency of $\mathrm{BBH}$ microcapsules was found to be $98.92 \pm 0.03 \%$ with a drug loading of $3.59 \pm 0.01 \%$. Bioadhesion percentage of coated microspheres was $91.23 \% \pm 8.2 \%$, while that of uncoated microspheres was only $21.3 \% \pm 5.7 \%$. The stability results demonstrated that there was no significant change in the appearance and $\mathrm{BBH}$ content after 10 days of exposure to $60^{\circ} \mathrm{C}, 92.5 \mathrm{RH}$, and $4500 \mathrm{~lx}$.

In vitro release behaviors of $\mathrm{BBH}$ from chitosan-coated alginate/gelatin microspheres and commercial tablets are shown in Figure 3. In simulated gastric fluid, about $17.62 \%$ of the drug loaded onto the microspheres was released in $0.5 \mathrm{~h}$. After 8 hours, the drug release ratio reached $71.29 \%$. The initial burst release was not apparent, which was related to cross-linked microspheres coated with chitosan. The release of $\mathrm{BBH}$ was faster in commercial tablets compared to $\mathrm{BBH}$ microspheres. About $71.2 \%$ of the drug was released in $10 \mathrm{~min}$ and $85.4 \%$ of the drug was released in $0.5 \mathrm{~h}$, followed by a constant release rate. 

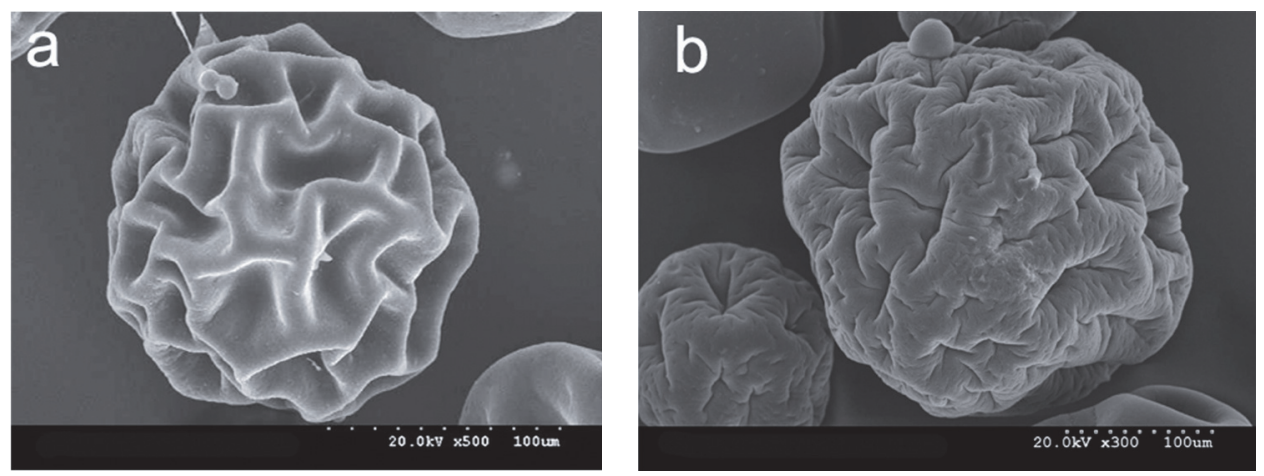

FIgURE 1: Scanning electron microscopy photographs of (a) alginate/gelatin BBH loaded microspheres and (b) chitosan-coated alginate/gelatin $\mathrm{BBH}$ loaded microspheres.

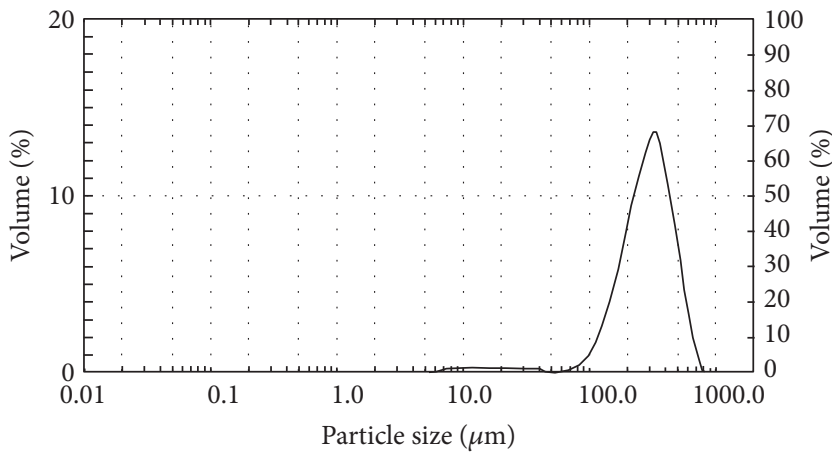

(a)

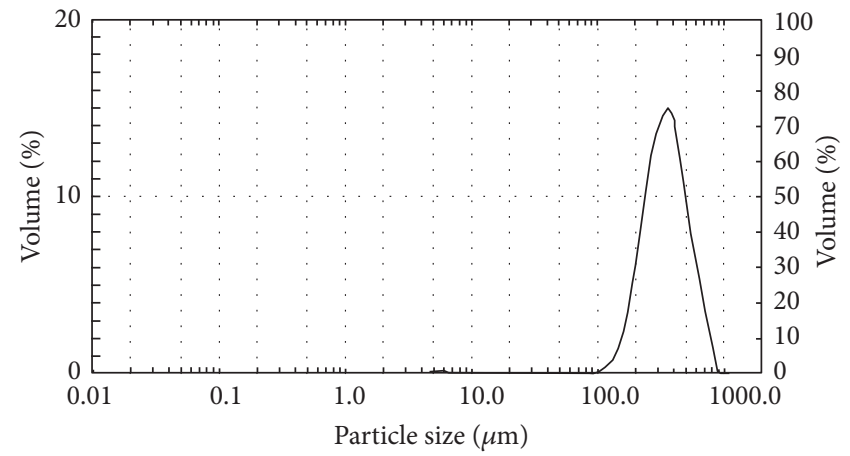

(b)

FIGURE 2: Size distribution of (a) alginate/gelatin BBH loaded microspheres and (b) chitosan-coated alginate/gelatin BBH loaded microspheres.

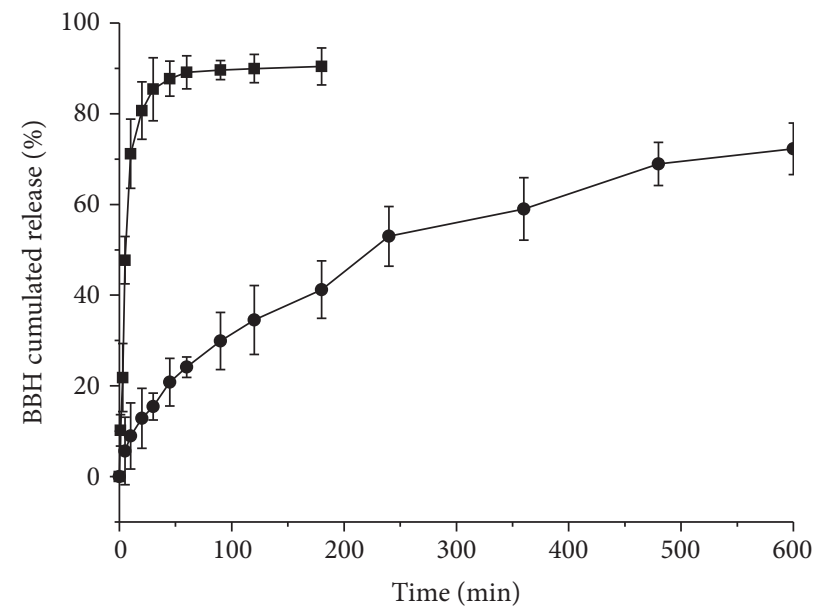

FIGURE 3: In vitro release testing of different $\mathrm{BBH}$ preparations at $37^{\circ} \mathrm{C}$ in simulated gastric fluid. The preparations tested were $\mathrm{BBH}$ commercial tablets (- - -) and chitosan-coated alginate/gelatin $\mathrm{BBH}$ loaded microspheres (-๑).

The LC-MS/MS method for the determination and quantitation of $\mathrm{BBH}$ in rat plasma was developed and validated. The mean recovery was from $102.1 \%$ to $104.1 \%$ for $0.5,5.0$, and $40 \mathrm{ng} / \mathrm{mL}$ of quality control samples with relative standard deviation (RSD) of less than $5 \%$. The intra- and interday precisions were within $3.0 \%$ and $3.5 \%$, respectively, with accuracy from $99.1 \%$ to $103.1 \%$. The RSD of repeatability was less than $4.1 \%$. The calibration curve was $Y=0.341 X+$ $0.00741(r=0.9995, n=6)$. The linear range for the determination of $\mathrm{BBH}$ was $0.2-50 \mathrm{ng} / \mathrm{mL}$, and limit of detection $(\mathrm{S} / \mathrm{N}>3)$ was $0.2 \mathrm{ng} / \mathrm{mL}$.

The plasma concentration-time profiles of $\mathrm{BBH}$ microspheres and commercial tablets are shown in Figure 4. When commercial tablets were given, $\mathrm{BBH}$ was undetectable at $24 \mathrm{~h}$. $\mathrm{BBH}$ microspheres exhibited a sustained release of $\mathrm{BBH}$ over $48 \mathrm{~h}$ postfeeding. The drug from microspheres was eliminated slowly compared to that from commercial tablets. The pharmacokinetic parameters, $C_{\max }, T_{\max }$, and AUC values were $6.5 \pm 0.7 \mathrm{ng} / \mathrm{mL}, 2 \pm 0.46 \mathrm{~h}$, and $109.4 \pm 0.8 \mathrm{ng} \cdot \mathrm{h} / \mathrm{mL}$, respectively, for $\mathrm{BBH}$ microspheres and $7.7 \pm 0.2 \mathrm{ng} / \mathrm{mL}, 1 \pm$ $0.37 \mathrm{~h}$, and $43.3 \pm 0.3 \mathrm{ng} \cdot \mathrm{h} / \mathrm{mL}$, respectively, for commercial tablets. $C_{\max }$ and $T_{\max }$ of $\mathrm{BBH}$ microspheres were smaller and higher than commercial tablets, respectively. The AUC of $\mathrm{BBH}$ microspheres was higher than the commercial tablets by $152.5 \%$, and the bioavailability of $\mathrm{BBH}$ microspheres was 2.52 times as much as the commercial tablets. Hence, $\mathrm{BBH}$ microspheres might be a promising sustained-release system for the oral delivery of $\mathrm{BBH}$.

\section{Discussion}

In the present study, water-in-oil emulsion technique was used to prepare $\mathrm{BBH}$ loaded microspheres. In microsphere 


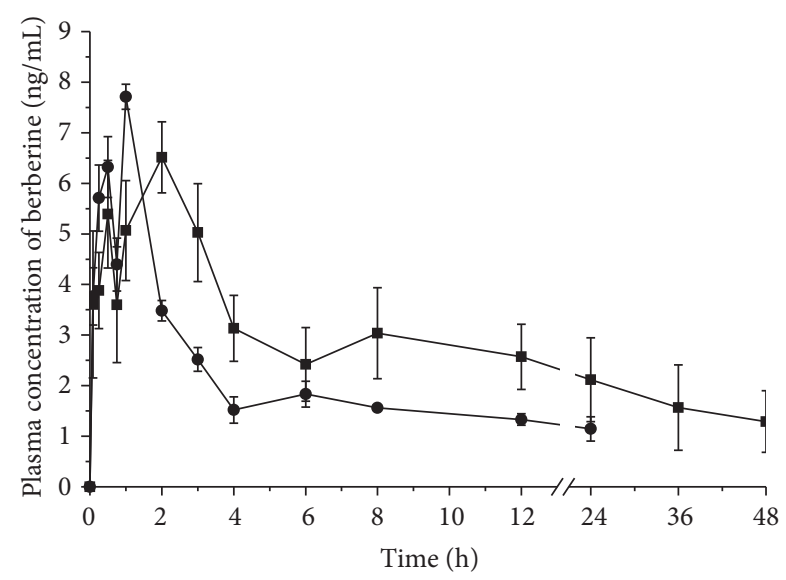

FIGURE 4: Plasma concentration profiles of berberine hydrochloride $(\mathrm{BBH})$ after oral administration of chitosan-coated alginate/gelatin BBH loaded microspheres (-口-) and BBH commercial tablets (-๑-) in rats $(n=4$ and $24 \mathrm{mg} / \mathrm{kg})$.

preparation, $\mathrm{W} / \mathrm{O}$ ratio is a key parameter to be considered to obtain good quality microspheres [30]. The $\mathrm{W} / \mathrm{O}$ ratio of higher than 10/1 results in excessive hydrogelation, whereas $\mathrm{W} / \mathrm{O}$ ratio less than $1 / 10$ induces adhesion microspheres. The claimed optimal ratio was between $2 / 25$ and $2 / 5$. To minimize the burst effect of $\mathrm{BBH}$, it would have been preferable to use high $\mathrm{W} / \mathrm{O}$ ratio according to a reported literature [31]. However, a high $\mathrm{W} / \mathrm{O}$ ratio often results in nonspherical particles, highly variable in size, and large particle size. Thus, a relatively high $\mathrm{W} / \mathrm{O}$ ratio of $1 / 5$ was selected in this study. Emulsifiers also play an important role in the high-quality microsphere preparation [32]. Hence, the influence of the type of emulsifier was evaluated with sodium desoxycholate, polyvinyl alcohol, Tween 80, and Span 80. Mixed emulsifier of Tween 80 and Span 80 produced less heterogeneous and less disrupt microspheres. This could be due to the variation in the hydrophilic-lipophilic balance as well as the nature of the surfactant.

So far, many chitosan-coated particles have been reported for oral drug delivery. The first improvement of the encapsulation process over our previous technique [33] was the enhancement of reproducibility, which remains a major issue for microsphere manufacturing process [34]. To obtain reproducible batches, the emulsion was made in a temperature-controlled apparatus, as it was very clear that emulsion temperature was a key process parameter determining the overall quality of the microspheres and thus the release patterns [35]. Hence, in the early stage of emulsion, high temperature was used to aid in the uniform dispersion of aqueous phase, which thus accelerates preparation of emulsion system. Later, the emulsion system was transferred to an ice-water bath to enhance shrinking droplets and to reduce the probability of collision and integration of dispersal droplets. Meanwhile, it also reduces the solubility of alginate and gelatin and thus improves the gelation of alginate and gelatin. In view of thermodynamics, low temperature was disadvantageous to obtain long-term stable emulsion system.
Hence, dispersant agent isopropanol was added to the emulsion system to further promote the gelation and increase the dispersibility of emulsion system. Then, cross-linking agent was added to solidify the microspheres. Another strategy to obtain reproducible batches was the vigorous stirring of the emulsion at $400 \mathrm{rpm}$, which allowed a homogeneous emulsion and thus high-quality microsphere batches. The second major improvement of prepared microspheres was the surface with deep elevation which could produce larger interfacial surface area than smooth surface and thus significantly enhance absorption.

The bioadhesion percentage of coated $\mathrm{BBH}$ loaded microspheres was higher than that of uncoated BBH loaded microspheres. This indicated that coated microspheres showed better bioadhesion properties than uncoated microspheres. Uncoated microspheres were entrapped in mucosa primarily because of their irregular shape unlike the spherical glass beads.

The release behaviors of $\mathrm{BBH}$ from chitosan-coated alginate/gelatin microspheres suggested that the drug molecules were trapped inside the cross-linked shells and only $17.6 \%$ of $\mathrm{BBH}$ was released within $30 \mathrm{~min}$. If it was a case of release from the surface, most of the adsorbed drug molecules could be released within 10 min when the microspheres came into contact with the release medium $[13,36]$. To restrict the burst effect, several methods were used. First, BBH was purified by recrystallization and then crushed into powder, because optimized milling process could have significant effect on the release profile [37] in particular on the burst effect. Second, drug loading method was optimized, as it could influence the burst effect [30]. Indeed, if saturated aqueous solution of $\mathrm{BBH}$ was used as the aqueous phase, drug loading of microspheres might reach up to $9.12 \%$. However, the microspheres showed a significant burst effect. This could be related to a percolation mechanism as previously reported for microparticles loaded with hydrophilic crystals such as cisplatin, at sufficient amounts (>18\% w/w) [38].

The pharmacokinetic parameters suggested that the bioavailability of BBH was significantly increased in chitosancoated alginate/gelatin microspheres as compared to commercial tablets. The results demonstrated that chitosancoated alginate/gelatin $\mathrm{BBH}$ loaded microspheres had a better sustained-release profile and better promoted oral absorption of $\mathrm{BBH}$ than commercial tablets. This is attributed to the bioadhesive property of chitosan and the advantages of microspheres. Thus, we conclude that a chitosan-coated alginate/gelatin delivery system, that is, a carrier combining alginate/gelatin microspheres and chitosan, is a favorable option for oral administration of $\mathrm{BBH}$.

\section{Conclusions}

Spherical and uniform-sized chitosan-coated alginate/gelatin microspheres with a mean diameter of $368.2 \mu \mathrm{m}$, drug loading of $3.59 \pm 0.01 \%$, and in situ bioadhesion percentage of $91.23 \% \pm 8.2 \%$ can be prepared using W/O emulsion method. In vitro dissolution experiments revealed that the dissolution of $\mathrm{BBH}$ from microspheres was slower than commercial tablets, and microspheres slowly released the 
$\mathrm{BBH}$ over $8 \mathrm{~h}$ duration. Meanwhile, in vivo studies revealed that the bioavailability of $\mathrm{BBH}$ microspheres as compared to commercial tablets was $252.5 \%$. Cumulatively, the results suggested that $\mathrm{BBH}$ microspheres could be used as a possible alternative to traditional oral formulations of $\mathrm{BBH}$ to improve its bioavailability.

\section{Competing Interests}

The authors declare that they have no competing interests.

\section{References}

[1] Y. S. Lee, W. S. Kim, K. H. Kim et al., "Berberine, a natural plant product, activates AMP-activated protein kinase with beneficial metabolic effects in diabetic and insulin-resistant states," Diabetes, vol. 55, no. 8, pp. 2256-2264, 2006.

[2] N. Shen, X. Li, T. Zhou et al., "Shensong Yangxin Capsule prevents diabetic myocardial fibrosis by inhibiting TGF- $\beta 1 / \mathrm{Smad}$ signaling," Journal of Ethnopharmacology, vol. 157, pp. 161-170, 2014.

[3] S. K. Kulkarni and A. Dhir, "On the mechanism of antidepressant-like action of berberine chloride," European Journal of Pharmacology, vol. 589, no. 1-3, pp. 163-172, 2008.

[4] S. K. Kulkarni and A. Dhir, "Possible involvement of L-argininenitric oxide (NO)-cyclic guanosine monophosphate (cGMP) signaling pathway in the antidepressant activity of berberine chloride," European Journal of Pharmacology, vol. 569, no. 1-2, pp. 77-83, 2007.

[5] W.-H. Peng, K.-L. Lo, Y.-H. Lee, T.-H. Hung, and Y.-C. Lin, "Berberine produces antidepressant-like effects in the forced swim test and in the tail suspension test in mice," Life Sciences, vol. 81, no. 11, pp. 933-938, 2007.

[6] W. Kong, J. Wei, P. Abidi et al., "Berberine is a novel cholesterollowering drug working through a unique mechanism distinct from statins," Nature Medicine, vol. 10, no. 12, pp. 1344-1351, 2004.

[7] G.-Y. Pan, G.-J. Wang, X.-D. Liu, J. P. Fawcett, and Y.-Y. Xie, "The involvement of P-glycoprotein in berberine absorption," Pharmacology \& Toxicology, vol. 91, no. 4, pp. 193-197, 2002.

[8] H.-J. Maeng, H.-J. Yoo, I.-W. Kim, I.-S. Song, S.-J. Chung, and C.-K. Shim, "P-glycoprotein-mediated transport of berberine across Caco-2 cell monolayers," Journal of Pharmaceutical Sciences, vol. 91, no. 12, pp. 2614-2621, 2002.

[9] Y. Zhang, Y.-L. Cui, L.-N. Gao, and H.-L. Jiang, "Effects of $\beta$-cyclodextrin on the intestinal absorption of berberine hydrochloride, a P-glycoprotein substrate," International Journal of Biological Macromolecules, vol. 59, pp. 363-371, 2013.

[10] J.-X. Zhu, D. Tang, L. Feng et al., "Development of selfmicroemulsifying drug delivery system for oral bioavailability enhancement of berberine hydrochloride," Drug Development and Industrial Pharmacy, vol. 39, no. 3, pp. 499-506, 2013.

[11] N. Foster and B. H. Hirst, "Exploiting receptor biology for oral vaccination with biodegradable particulates," Advanced Drug Delivery Reviews, vol. 57, no. 3, pp. 431-450, 2005.

[12] L. Illum, I. Jabbal-Gill, M. Hinchcliffe, A. N. Fisher, and S. S. Davis, "Chitosan as a novel nasal delivery system for vaccines," Advanced Drug Delivery Reviews, vol. 51, no. 1-3, pp. 81-96, 2001.
[13] X. Peng, L. Zhang, and J. F. Kennedy, "Release behavior of microspheres from cross-linked N-methylated chitosan encapsulated ofloxacin," Carbohydrate Polymers, vol. 65, no. 3, pp. 288-295, 2006.

[14] L.-Y. Wang, Y.-H. Gu, Q.-Z. Zhou, G.-H. Ma, Y.-H. Wan, and Z.-G. Su, "Preparation and characterization of uniformsized chitosan microspheres containing insulin by membrane emulsification and a two-step solidification process," Colloids and Surfaces B: Biointerfaces, vol. 50, no. 2, pp. 126-135, 2006.

[15] M. N. Uddin, N. J. Patel, T. Bhowmik et al., "Enhanced bioavailability of orally administered antisense oligonucleotide to nuclear factor kappa B mRNA after microencapsulation with albumin," Journal of Drug Targeting, vol. 21, no. 5, pp. 450-457, 2013.

[16] L.-D. Hu, Q.-B. Xing, C. Shang et al., "Preparation of rosiglitazone maleate sustained-release floating microspheres for improved bioavailability," Pharmazie, vol. 65, no. 7, pp. 477-480, 2010.

[17] M. D. Blanco, M. V. Bernardo, R. L. Sastre, R. Olmo, E. Muñiz, and J. M. Teijón, "Preparation of bupivacaine-loaded poly $(\varepsilon-$ caprolactone) microspheres by spray drying: drug release studies and biocompatibility," European Journal of Pharmaceutics and Biopharmaceutics, vol. 55, no. 2, pp. 229-236, 2003.

[18] R. K. Jha, S. Tiwari, and B. Mishra, "Bioadhesive microspheres for bioavailability enhancement of raloxifene hydrochloride: formulation and pharmacokinetic evaluation," AAPS PharmSciTech, vol. 12, no. 2, pp. 650-657, 2011.

[19] A. Gifani, M. Taghizadeh, A. A. Seifkordi, and M. Ardjmand, "Preparation and investigation the release behaviour of wax microspheres loaded with salicylic acid," Journal of Microencapsulation, vol. 26, no. 6, pp. 485-492, 2009.

[20] S. G. Gayakwad, N. K. Bejugam, N. Akhavein, N. A. Uddin, C. E. Oettinger, and M. J. D'Souza, "Formulation and in vitro characterization of spray-dried antisense oligonucleotide to NF-kappaB encapsulated albumin microspheres," Journal of Microencapsulation, vol. 26, no. 8, pp. 692-700, 2009.

[21] F. Yang, F.-L. Song, Y.-F. Pan et al., "Preparation and characteristics of interferon-alpha poly(lactic-co-glycolic acid) microspheres," Journal of Microencapsulation, vol. 27, no. 2, pp. 133141,2010

[22] S. S. Xu, J. Wu, and W. Jiang, "Synthesis and characterisation of a $\mathrm{pH}$-sensitive magnetic nanocomposite for controlled delivery of doxorubicin," Journal of Microencapsulation, vol. 32, no. 6, pp. 533-537, 2015.

[23] E. Başaran, E. Yenilmez, M. S. Berkman, G. Büyükköroğlu, and Y. Yazan, "Chitosan nanoparticles for ocular delivery of cyclosporine A," Journal of Microencapsulation, vol. 31, no. 1, pp. 49-57, 2014.

[24] N. Yüksel, A. Aydmli, Y. Özalp, and N. Özdemir, "Evaluation and preparation of controlled release lipid micropheres of sulphamethizole by a congealable disperse phase encapsulation method," Acta Poloniae Pharmaceutica-Drug Research, vol. 57, no. 3, pp. 187-192, 2000.

[25] T. Chandy and C. P. Sharma, "Chitosan-as a biomaterial," Biomaterials, Artificial Cells and Artificial Organs, vol. 18, no. 1, pp. 1-24, 1990.

[26] W.-M. Hou, S. Miyazaki, M. Takada, and T. Komai, "Sustained release of indomethacin from chitosan granules," Chemical \& Pharmaceutical Bulletin, vol. 33, no. 9, pp. 3986-3992, 1985.

[27] H.-W. Sung, D.-M. Huang, W.-H. Chang, R.-N. Huang, and J.C. Hsu, "Evaluation of gelatin hydrogel crosslinked with various 
crosslinking agents as bioadhesives: in vitro study," Journal of Biomedical Materials Research, vol. 46, no. 4, pp. 520-530, 1999.

[28] X. Z. Shu and K. J. Zhu, "A novel approach to prepare tripolyphosphate/chitosan complex beads for controlled release drug delivery," International Journal of Pharmaceutics, vol. 201, no. 1, pp. 51-58, 2000.

[29] K. V. R. Rao and P. Buri, "A novel in situ method to test polymers and coated microparticles for bioadhesion," International Journal of Pharmaceutics, vol. 52, no. 3, pp. 265-270, 1989.

[30] F. Lagarce, P. Renaud, N. Faisant et al., "Baclofen-loaded microspheres: preparation and efficacy testing in a new rabbit model," European Journal of Pharmaceutics and Biopharmaceutics, vol. 59, no. 3, pp. 449-459, 2005.

[31] F. T. Meng, G. H. Ma, W. Qiu, and Z. G. Su, "W/O/W double emulsion technique using ethyl acetate as organic solvent: effects of its diffusion rate on the characteristics of microparticles," Journal of Controlled Release, vol. 91, no. 3, pp. 407-416, 2003.

[32] H. Jeffery, S. S. Davis, and D. T. O'Hagan, “The preparation and characterization of poly(lactide-co-glycolide) microparticles. II. The entrapment of a model protein using a (water-in-oil)-inwater emulsion solvent evaporation technique," Pharmaceutical Research, vol. 10, no. 3, pp. 362-368, 1993.

[33] Q.-S. Wang, Y.-L. Cui, Y. Zhang, Y.-B. Zhang, and X.-M. Gao, "Preparation and evaluation of chitosan-coated alginate/ gelatin sustained releasing microspheres containing berberine hydrochloride in vitro," in Proceedings of the 3rd International Conference on Bioinformatics and Biomedical Engineering (iCBBE '09), pp. 1-4, IEEE, Beijing, China, June 2009.

[34] J. Herrmann and R. Bodmeier, "Biodegradable, somatostatin acetate containing microspheres prepared by various aqueous and non-aqueous solvent evaporation methods," European Journal of Pharmaceutics and Biopharmaceutics, vol. 45, no. 1, pp. 75-82, 1998.

[35] Y.-Y. Yang, H.-H. Chia, and T.-S. Chung, "Effect of preparation temperature on the characteristics and release profiles of PLGA microspheres containing protein fabricated by doubleemulsion solvent extraction/evaporation method," Journal of Controlled Release, vol. 69, no. 1, pp. 81-96, 2000.

[36] P. He, S. S. Davis, and L. Illum, "Chitosan microspheres prepared by spray drying," International Journal of Pharmaceutics, vol. 187, no. 1, pp. 53-65, 1999.

[37] A. Gèze, M. C. Venier-Julienne, D. Mathieu, R. Filmon, R. Phan-Tan-Luu, and J. P. Benoit, "Development of 5-iodo-2' deoxyuridine milling process to reduce initial burst release from PLGA microparticles," International Journal of Pharmaceutics, vol. 178, no. 2, pp. 257-268, 1999.

[38] G. Spenlehauer, M. Vert, J.-P. Benoît, F. Chabot, and M. Veillard, "Biodegradable cisplatiim microspheres prepared by the solvent evaporation method: morphology and release characteristics," Journal of Controlled Release, vol. 7, no. 3, pp. 217-229, 1988. 

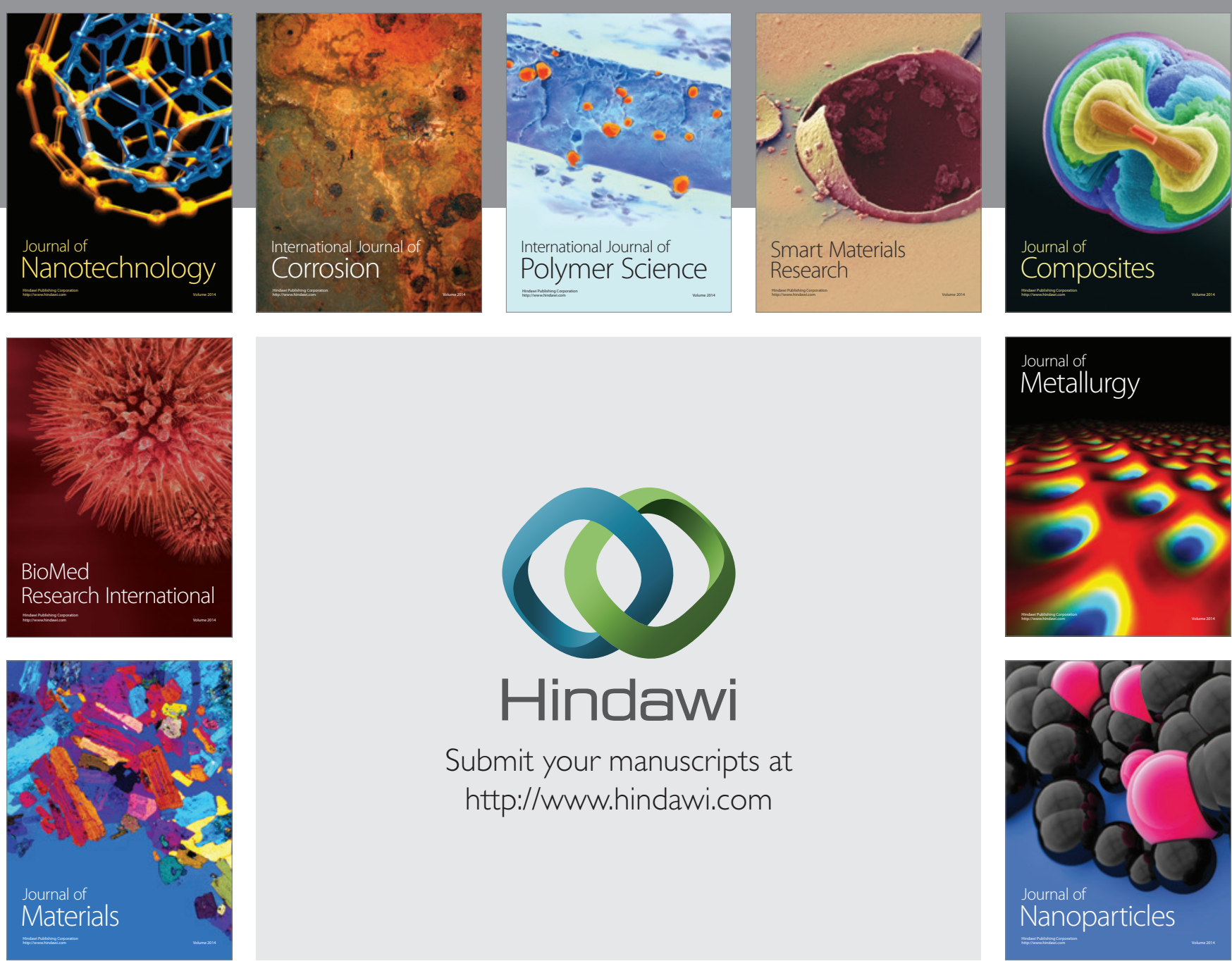

\section{Hindawi}

Submit your manuscripts at

http://www.hindawi.com

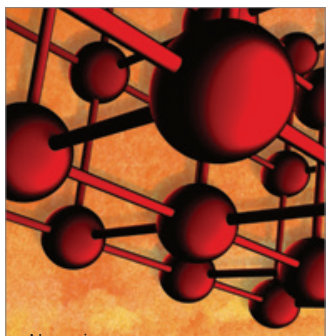

Materials Science and Engineering
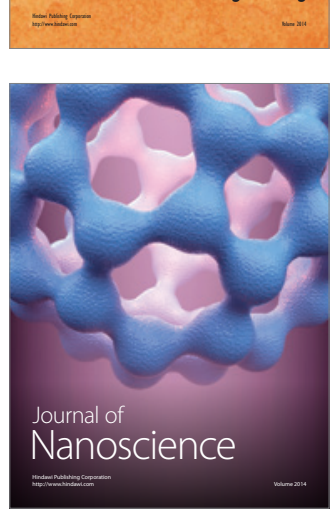
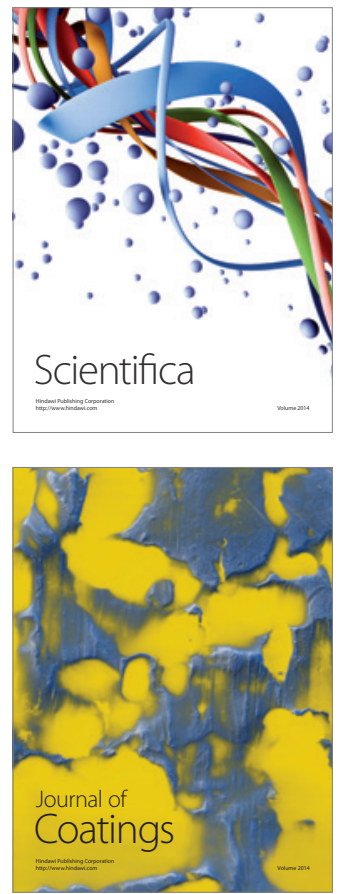
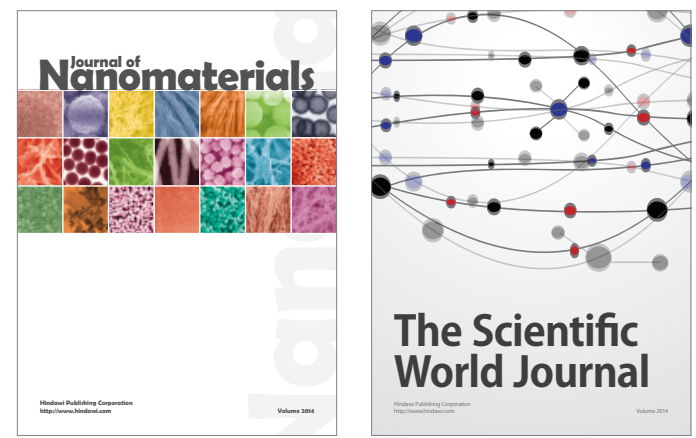

The Scientific World Journal
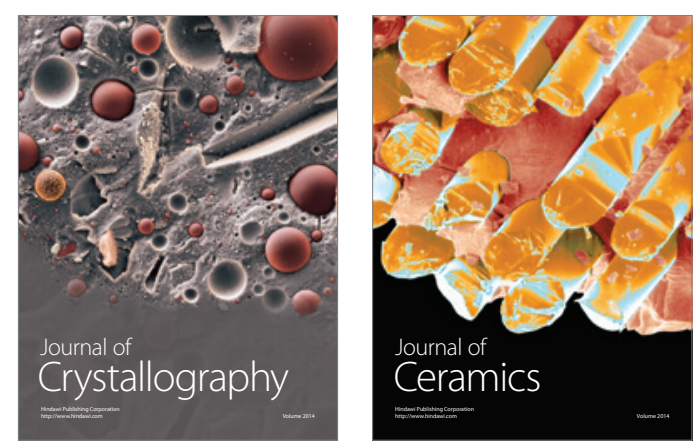
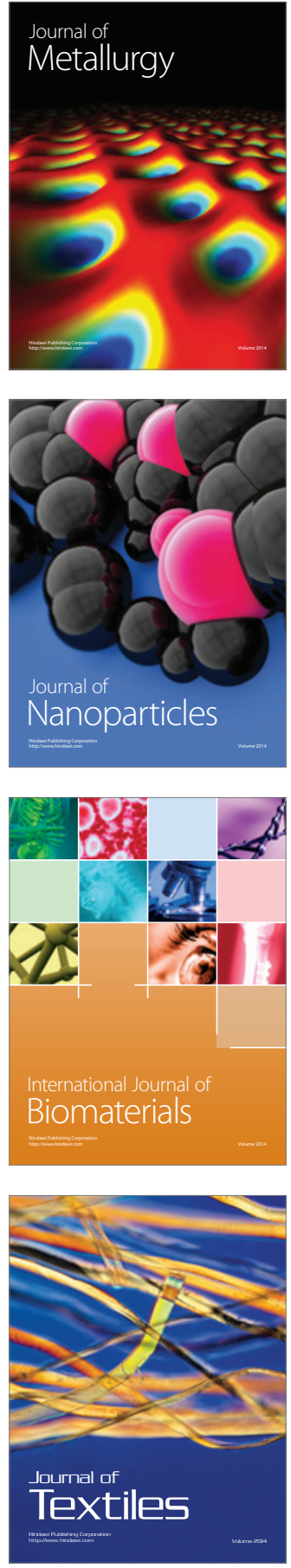